\title{
Local and systemic control of the osteoblast
}

\section{Commentary}

\author{
Gordon J. Strewler
}

Veterans Affairs Boston Healthcare System, Brigham and Women's Hospital and Harvard Medical School, Boston, Massachusetts, USA

Address correspondence to: Gordon J. Strewler, Veterans Affairs Boston Healthcare System, 1400 VFW Parkway, West Roxbury, Massachusetts 02132, USA. Phone: (617) 363-5559; Fax: (617) 363-5549; E-mail: Gordon.Strewler@med.va.gov.

The role of parathyroid hormone (PTH) as a protector of extracellular calcium levels requires that, in response to the challenge of hypocalcemia, the hormone activate a mechanism for the release of calcium from bone, its principal body storehouse. PTH does so not by a direct action on the bone-resorbing cell, the osteoclast, but by an indirect effect mediated by PTH receptors on bone stromal cells, including the mature bone-forming cell, the osteoblast. Regarded from the perspective of calcium homeostasis, the entrainment of osteoblasts and their stromal precursors by PTH could serve the straightforward function of linking new bone formation to bone resorption, thus restoring bone sacrificed to the demands of hypocalcemia. For example, primary hyperparathyroidism is an osteoporotic state, but most of the bone loss occurs from the cortical compartment, which is impoverished of osteoblasts. The cancellous or trabecular compartment of bone, which presents a large surface area to osteoblastic new bone formation, is relatively preserved in primary hyperparathyroidism and may actually be increased (1).

However, the anabolic effect of PTH is markedly accentuated when the hormone is administered once daily, an ancient observation (2) that has now led to the introduction of therapy using synthetic PTH fragments for the treatment of osteoporosis. Daily subcutaneous administration of PTH induces rapid, dramatic effects in cancellous bone mass (3), and a preliminary report indicates a marked reduction in the incidence of new fractures in PTHtreated postmenopausal women (4). Because its antifracture effect works through an anabolic mechanism, PTH offers a potentially important alternative to existing agents for the prevention of osteoporotic fractures, all of which (estrogens, the selective estrogen response modifier raloxifene and bis- phosphonates) have a primarily antiresorptive effect (5). For example, PTH could ultimately prove to be the therapy of choice for fracture prevention in individuals who have already experienced extensive bone loss.

The role of the PTH-receptor system in bone is likely to be complex. Bone also possesses an endogenous ligand of the PTH receptor, the PTH-related protein (PTHrP), which is expressed in both cartilage and osteoblasts of bone. In cartilage, PTHrP functions downstream of Indian hedgehog in a pathway that regulates the exit of chondrocytes from a proliferating pool into a terminally differentiated cell type, the hypertrophic chondrocyte, which lays down a matrix capable of mineralization (6). Ablation of the PTHrP gene produces a neonatally fatal chondrodystrophy (7). Unfortunately, this knockout does not clarify the role of PTHrP in steady-state bone metabolism because of this early lethality and profoundly disturbed endochondral bone formation.

In this issue of the JCI, Calvi et al. probe the role of the receptor for $\mathrm{PTH}$ and PTHrP by expressing a constitutively active mutant form of the receptor in bone (8). The activating mutation of the $\mathrm{PTH} / \mathrm{PTHrP}$ receptor was identified in a human disorder called Janssen's metaphyseal chondrodysplasia (9), in which patients have lifelong hypercalcemia with suppression of PTH, presumably as a result of constitutive activity of the receptor in bone and kidney. Calvi et al. targeted the activated PTH/PTHrP receptor mutant to the osteoblast lineage using the promoter from the gene for $\alpha 1(\mathrm{I})$ collagen. In one founder mouse line there was a striking bone phenotype - a marked increase in trabecular bone volume, attributable to increased numbers of osteoblasts and an increase in the rate of matrix mineralization per cell. The overall effect of expressing the constitutively active PTH/PTHrP receptor in trabecular bone resembles that of daily PTH administration. Ablation of the PTH/PTHrP receptor has the converse effect, markedly decreasing trabecular number (10).

In contrast to the increase in trabecular bone, cortical bone thickness is markedly reduced in mice expressing the constitutively active $\mathrm{PTH} / \mathrm{PTHr} \mathrm{P}$ receptor. This cortical bone loss can be attributed to the virtual absence of bone apposition on the periosteal surface, with preservation of endosteal bone formation. Cortical bone in these animals is porous, with increased numbers of osteoclasts, demonstrating directly that, in keeping with the classic model, activation of the $\mathrm{PTH}$ receptor in the osteoblast lineage is sufficient to activate osteoclastic bone resorption. Although they are normocalcemic, transgenic mice show reduced serum PTH levels, consistent with an autonomous increase in calcium resorption from bone. The mice thus reproduce the overall pattern of bone loss in primary hyperparathyroidism, with increased bone turnover, predominately cortical destruction and preservation of cancellous bone.

Increased trabecular bone formation in hyperparathyroid states mainly results from increased numbers of osteoblasts, but the genesis of these osteoblasts remains to be clarified. The work of Calvi et al. (8) is consistent with both of the commonly cited hypotheses to explain such a finding, since they found both an increase in proliferation within the osteoblast lineage and a reduction in the rate of osteoblast apoptosis (11). Not addressed is a third possibility, that at least some of the osteoblasts that populate bone surfaces in hyperparathyroid states arise from activation of bone-lining cells, the quiescent cells on inactive bone surfaces (12). Quantitative approaches to cell kinetics in the osteoblast lineage will ultimately be necessary to resolve what are likely to be multiple receptor-mediated effects of PTH. If designed so as to 
include periosteal cells, such studies may also disclose what differences between periosteal and endosteal cells lead to opposite effects of the active $\mathrm{PTH}$ receptor on bone formation.

Constitutive $\mathrm{PTH} / \mathrm{PTHrP}$ receptor activity is also associated with large amounts of marrow fibrosis. Marrow fibrosis is seen in severe primary hyperparathyroidism (osteitis fibrosa cystica) and also in fibrous dysplasia of bone, a condition that results from constitutive activity of a downstream component of the PTH receptor complex, the G protein $\mathrm{G}_{\mathrm{s}} \alpha$. Calvi et al. (8) make the important observation that fibrous cells in bone marrow heterogeneously express a number of osteoblast markers and may therefore represent an expanded pool of osteoblast precursors. However, some or all of the cells may be arrested at an early stage of differentiation, as appears to be the case with marrow cells that express a constitutively active form of $\mathrm{G}_{\mathrm{s}} \alpha$ (13), and would therefore fail to populate the bone surface.

Calvi et al. (8) show that constitutive activation of the $\mathrm{PTH} / \mathrm{PTHrP}$ receptor in bone enhances trabecular bone mass and decreases cortical bone mass. Interestingly, ablation of the receptor has opposite effects in both bone compartments (with the caveat that the observations were made in late-stage mouse embryos with a severe chondrodysplasia) (10). Considering these results, does the receptor function in the physiological regulation of the osteoblast? And if so, which ligand of the receptor is more important?

Hypoparathyroidism is associated with a low-turnover bone state with preservation of the trabecular bone mass. This is quite different from the phenotype of the receptor knockout, raising the possibility that PTH is dis- pensable in physiological regulation of trabecular osteoblasts or that this ligand acts through some other receptor to diminish bone mass. Mice heterozygous for a null mutation of the PTHrP gene, conversely, show reduced trabecular bone volume (14), although this effect may be explained in part by their residual mild chondrodysplasia. It is thus conceivable that PTHrP functions in bone in a manner somewhat like cartilage: PTHrP, expressed in the mature osteoblast, could regulate entry of receptor-expressing osteoblast precursors into the compartment of boneforming cells, or their exit from this compartment through apoptosis. These issues can be clarified by tissue-specific ablation or substitution of the PTHrP and $P$ THrP receptor genes in bone, using methods such as cre-lox technology.

As the work of Calvi et al. (8) suggests, the osteoblast is now emerging as a cell under independent local control (15), with the PTH, PTHrP, and their receptor offering candidates for local regulation and possible therapeutic targets. New modes of systemic regulation of the osteoblast have also emerged. The finding that leptin-deficient mice have increased bone mass (16) not only provides an explanation for the protection of obesity against osteoporosis, but also identifies a neural control circuit for regulation of the osteoblast (15). Because postmenopausal osteoporosis is characterized by high levels of bone resorption, investigation into its cause and therapy has hitherto focused on the osteoclast, but, for reasons that remain poorly understood, osteoblast function is also impaired in this condition (5). With the advent of PTH as an osteoblast-targeted therapeutic, it seems certain that the osteoblast will remain a focus of osteoporosis research in years to come.
1. Parisien, M., et al. 1990. The histomorphometry of bone in primary hyperparathyroidism: preservation of cancellous bone structure. J. Clin. Endocrinol. Metab. 70:930-938.

2. Bauer, W., Aub, J., and Albright, F. 1929. Studies in calcium phosphorus metabolism: study of bone trabeculae as ready available reserve supply of calcium. J. Exp. Med. 49:145-162

3. Lane, N.E., et al. 1998. Parathyroid hormone treatment can reverse corticosteroid-induced osteoporosis. Results of a randomized controlled clinical trial. J. Clin. Invest. 102:1627-1633.

4. Neer, R., et al. 2000. Recombinant human PTH [rhPTH 1-34] reduces the risk of spine and nonspine fractures in postmenopausal osteoporosis. 82nd Annual Meeting, The Endocrine Society.

5. Rodan, G.A., and Martin, T.J. 2000. Therapeutic approaches to bone diseases. Science. 289:1508-1514.

6. Strewler, G.J. 2000. The physiology of parathyroid hormone-related protein. N. Engl. J. Med. 342:177-185.

7. Karaplis, A.C., et al. 1994. Lethal skeletal dysplasia from targeted disruption of the parathyroid hormone-related peptide gene. Genes Dev. 8:277-289.

8. Calvi, L.M., et al. 2000. Activated parathyroid hormone/parathyroid hormone-related protein receptor in osteoblastic cells differentially affects cortical and trabecular bone. J. Clin. Invest. 107:277-286.

9. Schipani, E., et al. 1996. Constitutively activated receptors for parathyroid hormone and parathy roid hormone-related peptide in Jansen's metaphyseal chondrodysplasia. N. Engl. J. Med. 335:708-714.

10. Lanske, B., et al. 1999. Ablation of the PTHrP gene or the PTH/PTHrP receptor gene leads to distinct abnormalities in bone development. J. Clin. Invest. 104:399-407.

11. Jilka, R.L., et al. 1999. Increased bone formation by prevention of osteoblast apoptosis with parathyroid hormone. J. Clin. Invest. 104:439-446.

12. Dobnig, H., and Turner, R.T. 1995. Evidence that intermittent treatment with parathyroid hormone increases bone formation in adult rats by activation of bone lining cells. Endocrinology. 136:3632-3638.

13. Bianco, P., et al. 1998. Reproduction of human fibrous dysplasia of bone in immunocompromised mice by transplanted mosaics of norma and Gsalpha-mutated skeletal progenitor cells. J. Clin. Invest. 101:1737-1744.

14. Amizuka, N., et al. 1996. Haploinsufficiency of parathyroid hormone-related peptide (PTHrP) results in abnormal postnatal bone development. Dev. Biol. 175:166-176.

15. Ducy, P., Schinke, T., and Karsenty, G. 2000. The osteoblast: a sophisticated fibroblast under central surveillance. Science. 289:1501-1504.

16. Ducy, P., et al. 2000. Leptin inhibits bone formation through a hypothalamic relay: a central control of bone mass. Cell. 100:197-207. 